\title{
Multiple-Input Multiple-Output OFDM with Index Modulation Using Frequency Offset
}

\author{
Poonam Ganesh. Swami ${ }^{1}$, Prof.S.K.Sudhansu ${ }^{2}$ \\ ${ }^{I}(P G$ Department, M.B.E.Society's College of Engineering, Ambajogai India) \\ ${ }^{2}((P G$ Department, M.B.E.Society's College of Engineering, Ambajogai India)
}

\begin{abstract}
With increasing demand of high datarate applications at low cost, wireless communication is the key area of research, the solution to this problem is OFDM (Orthogonal Frequency Division Multiplexing). Multicarrier transmission technique like OFDM with IM (Index Modulation) is the best choice over classical OFDM. Sending the information at the indices of active subcarriers is extra information source. In this work we propose MIMO OFDM IM using frequency offset by combining MIMO (Multiple Input Multiple Output) \& OFDM IM transmission techniques. The different low complexity transceiver structure of MIMO OFDM IM is developed \& shown via numerical calculations on Matlab platform. Here we propose MIMO OFDM IM using frequency offset that achieves significantly better BER (Bit Error Rate) performance than MIMO OFDM IM for several different system configurations.
\end{abstract}

Keywords : OFDM, MIMO, index modulation (IM), MMSE, and frequency offset.

\section{Introduction}

For high speed wireless communication OFDM is the best choice \& very famous multicarrier signaling format \& used in many standards such as Long Term Evolution (LTE), wireless LAN (Local Area Network) Digital Audio \& Video Broadcasting (DAB \& DVB).IEEE 802.11. The combination of OFDM with MIMO system appears as an attractive alternative for 5G wireless networks [2]. In this OFDM IM, the information not only sends by M-ary constellation symbols but also by the indices of active subcarriers which are activated according to the incoming information bits [3]. Due to interesting trade off in error performance and spectral efficiency [6]-[12] subcarrier index modulation techniques for OFDM [3]-[5] gain considerable attention from researchers. The BER performance of OFDM IM is derived in [5]. By Selecting active subcarriers in more flexible way [7] its spectral efficiency is improved. In [8] \& [9] the selection of optimal number of active subcarriers in OFDM IM deals by authors. Recently use of coordinate interleaving with OFDM IM is discussed in [9]. In the proposed scheme [1] OFDM frames are transmitted by using OFDM transmission techniques and at receivers side these OFDM frames are demodulated using low complexity minimum mean square error (MMSE) \& LLR (Log Likelihood Ratio) based detector. By using frequency offset method we reduced BER of proposed system than previous MIMO OFDM IM [1].

The wideband frequency selective fading channel is divided into many narrow band sub channels. If no of sub channels are high each sub channel would be considered as a flat this is because we transmit many narrow band overlapping digital signals in parallel inside one wideband that's why it saves lots of bandwidth compared to FDM (frequency Division Multiplexing). In this paper we are considering the system model \& detection schemes as proposed in the previous woks $[1,2]$.we provide the performance analysis of given system using frequency offset \& present the performance analysis to reduce BER (Bit Error Rate) and results are obtained using extensive numerical calculations on MATLAB. The rest of the paper is organized as follows: In section II, we illustrate the given system model of MIMO OFDM IM. In section III, we discussed different detection schemes. In section IV, we present MIMO OFDM IM using frequency offset. In section V, MATLAB results are given. Finally section VI concludes the letter.

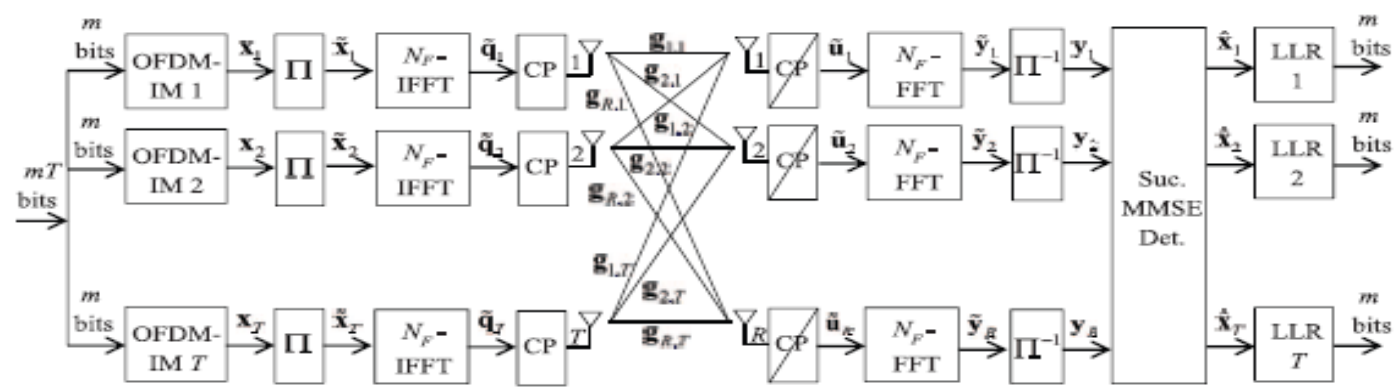

Fig1: Transceiver structure of MIMO OFDM IM 


\section{System Model Of Mimo Ofdm Im}

The MIMO OFDM IM transceiver structure is shown in Fig1. It employs $\mathrm{T}$ transmitter and R receiver antennas. A total $\mathrm{mT}$ information bits enter the MIMO OFDM IM. These $\mathrm{mT}$ bits are split into T groups \& $\mathrm{m}$ bits are processed in each branch of transmitter by OFDM index modulator these $\mathrm{m}$ bits are used to form $\mathrm{NF}^{*} 1$ OFDM IM block $x_{t}=\left[\mathrm{x}_{\mathrm{t}}(1) \mathrm{x}_{\mathrm{t}}(2) \ldots \mathrm{x}_{\mathrm{t}}(\mathrm{N})\right]^{T} ; \mathrm{t}=1,2 \ldots \mathrm{T}$ in each branch of the transmitter, where NF is the size of the fast Fourier transform (FFT) and $\mathrm{x}_{\mathrm{t}}\left(n_{f}\right) \in\{0, S\} ; n_{f}=1,2 \ldots \ldots N_{F}$. According to principle of OFDM IM these $\mathrm{m}$ bits are split into $\mathrm{G}$ groups [3] each containing $\mathrm{P}=P_{1}+P_{2}$ bits, which are used to form OFDM-IM sub blocks $X_{t}^{g}=\left[X_{t}^{g}(1) X_{t}^{g}(2) \ldots \ldots X_{t}^{g}(N)\right]^{T} ; \mathrm{g}=1,2 \ldots \mathrm{G}$ of length $\mathrm{N}=N_{F} / \mathrm{G}$, where $X_{t}^{g}(n) \in\{0, S\}, \mathrm{n}=1 \ldots \mathrm{N}$. We know that $P_{1}=\left\lfloor\log _{2}(C(N, K))\right\rfloor$ bits, only $\mathrm{K}$ out of $\mathrm{N}$ available subcarriers are set as an active and remaining N-K subcarriers are inactive (set to 0 ). On the other hand $P_{2}=$ $\mathrm{K} \log _{2}(\mathrm{M})$ bits are mapped onto M- ary signal constellation. Therefore unlike classical MIMO-OFDM, $X_{t}, \mathrm{t}=$ $1,2 \ldots$ T contains some zero terms whose positions carry information for MIMO-OFDM-IM [1]. Selection of index is performed by reference look up table. For $\mathrm{N}=4, \mathrm{~K}=3$ are given in table I \& II respectively.

Table I: $\mathrm{N}=4, \mathrm{~K}=2 \& P_{1}=2$

\begin{tabular}{|c|c|c|}
\hline Bits & Indices $\left(\mathbf{j}_{t}^{g}\right)^{7}$ & OFDM-IM subblocks $\left(\mathbf{x}_{t}^{g}\right)^{\mathrm{T}}$ \\
\hline$\left[\begin{array}{ll}0 & 0\end{array}\right]$ & {$\left[\begin{array}{ll}1 & 3\end{array}\right]$} & {$\left[\begin{array}{llll}s_{t}^{g}(1) & 0 & s_{t}^{g}(2) & 0\end{array}\right]$} \\
\hline$\left[\begin{array}{ll}0 & 1\end{array}\right]$ & $\begin{array}{|ll|}2 & 4 \\
\end{array}$ & \begin{tabular}{|llll}
0 & $s_{t}^{g}(1)$ & 0 & $s_{t}^{g}(2)$ \\
\end{tabular} \\
\hline$\left[\begin{array}{ll}1 & 0\end{array}\right]$ & $\left.\begin{array}{ll}1 & 4\end{array}\right]$ & $s_{t}^{g}(1) \quad 0 \quad 0 \quad s_{t}^{g}(2)$ \\
\hline$\left[\begin{array}{ll}1 & 1\end{array}\right]$ & $\left.\begin{array}{ll}2 & 3\end{array}\right]$ & $\left.\begin{array}{llll}0 & s_{t}^{g}(1) & s_{t}^{g}(2) & 0\end{array}\right]$ \\
\hline
\end{tabular}

Table II: N=4, $\mathrm{K}=3 \& P_{1}=2$

\begin{tabular}{|c|c|c|}
\hline Bits & Indices $\left(\mathbf{j}_{t}^{g}\right)$ & OFDM-IM subblocks $\left(\mathbf{x}_{t}^{g}\right)$ \\
\hline$\left[\begin{array}{ll}0 & 0\end{array}\right]$ & {$\left[\begin{array}{lll}1 & 2 & 3\end{array}\right]$} & $\begin{array}{llll}s_{t}^{g}(1) & s_{t}^{g}(2) & s_{t}^{g}(3) & 0\end{array}$ \\
\hline$\left[\begin{array}{ll}0 & 1\end{array}\right]$ & $\begin{array}{lll} & 2 & 4 \\
\end{array}$ & $\left.\begin{array}{lllll}s_{t}^{g}(1) & s_{t}^{g}(2) & 0 & s_{t}^{g}(3)\end{array}\right]$ \\
\hline$\left[\begin{array}{ll}1 & 0\end{array}\right]$ & $\begin{array}{|lll|}1 & 3 & 4 \\
\end{array}$ & $\begin{array}{lllll}s_{t}^{g}(1) & 0 & s_{t}^{g}(2) & s_{t}^{g}(3) \\
\end{array}$ \\
\hline$\left[\begin{array}{ll}1 & 1\end{array}\right]$ & {$\left[\begin{array}{lll}2 & 3 & 4\end{array}\right]$} & $\left.\begin{array}{llll}0 & s_{t}^{g}(1) & s_{t}^{g}(2) & s_{t}^{g}(3)\end{array}\right]$ \\
\hline
\end{tabular}

Different OFDM IM sub blocks are used to form main OFDM block $X_{t} ; \mathrm{t}=1,2 \ldots \ldots$ T. To transmit the elements from uncorrelated channels $\mathrm{G} * \mathrm{~N}$ block interleaves are used at transmitter. This interleaved frames are processed by IFFT to obtain $\widetilde{q}_{t}, \mathrm{t}=1 \ldots \mathrm{T}$. Assume time domain OFDM symbols are normalized to have unit energy, and then it makes use of different blocks such as cyclic prefix (CP), parallel to serial, digital to analog conversions. After words the resulting signal is transmitted over frequency selective Rayleigh fading MIMO channel. After removing cyclic prefix \& performing FFT operations in each branch of the receiver, the inputoutput relationship of MIMO OFDM IM scheme in frequency domain is obtained as [1]

$$
\widetilde{Y_{r}}=\sum_{t=1}^{T} \operatorname{diag}\left(\widetilde{X_{t}}\right) h_{r, t}+W_{r}
$$

For $\mathrm{r}=1 \ldots \mathrm{R}$, where $\widetilde{Y}_{r}=\left[\widetilde{Y}_{r}(1) \widetilde{Y}_{r}(2) \ldots . . \widetilde{Y}_{r}\left(N_{F}\right)\right]^{T}$ is the vector of the received signals for receiving antenna $\mathrm{r}, h_{r, t}$ is the frequency response of wireless channel between transmit $\mathrm{T} \&$ receiver $\mathrm{R} \& W_{r}$ is a vector of noise samples. The elements of $h_{r_{2}, t}$ and $W_{r}$ follow $C N(0,1)$ and $C N\left(0, N_{O, F}\right)$ distributions, respectively, where $\mathrm{N}_{\mathrm{O}, \mathrm{F}}$ denotes the variance of the noise samples in the frequency domain, which is related to the variance of the noise samples in the time domain as $\mathrm{N}_{0, \mathrm{t}}=(\mathrm{NF} /(\mathrm{KG})) \mathrm{N}_{0, \mathrm{f}}$.

\section{Detection Of Mimo-Ofdm-Im Scheme}

The received signals are obtained for receive antenna $r$, after block deinterleaving as,

$$
Y_{r}=\sum_{t=1}^{T} \operatorname{diag}\left(X_{t}\right) \widetilde{h_{r, t}}+\widetilde{W_{r}}
$$


Where $\widetilde{h_{r, t}}$ and $\overline{W_{r}}$ are deinterleaved versions of $h_{r_{n} t}$ and $W_{r}$ respectively. The detection of the MIMO OFDM-IM scheme can be performed by the separation of the received signals in (2) for each sub block $g=$ 1 ...G [1]

$$
Y_{r}^{g}=\sum_{t=1}^{T} \operatorname{diag}\left(X_{t}^{g}\right){\widetilde{h_{r, t}}}^{g}+{\overline{W_{r}}}^{g}
$$

For detection maximum likelihood (ML) detector is a straight forward but costly solution which can be realized for each sub block $\mathrm{g}$ as

$$
\hat{X}_{1}^{g}, \ldots \ldots \hat{X}_{T}^{g}=\arg \underbrace{\min }_{X_{1}^{g} \operatorname{mox}_{T}^{g}} \sum_{r=1}^{R}\left\|y_{r}^{g}-\sum_{t=1}^{T} \operatorname{diag}\left(X_{t}^{g}\right) \widetilde{h_{r, t}^{g}}\right\|^{2}
$$

Due to interference between sub blocks of different transmit antennas ML has a make a joint search over all transmit antennas, but because of complex multiplications which become impractical for higher order modulations \& MIMO systems that's why in this work we propose MMSE detection \& LLR calculation based detector, which has linear decoding complexity as that of classical MIMO OFDM \& MMSE. Data symbols can be simply recovered after processing received signal vector with MMSE detector. It is not possible to detect all the transmitted symbols for a given subcarrier therefore $\mathrm{N}$ successive MMSE detections are performed for proposed scheme. Unlike classical MIMO OFDM $\widehat{X_{t}^{\bar{g}}}$ contains some zero terms, whose positions carry information therefore independent detection of the data symbols is not a straight forward problem for proposed scheme as an example rounding off individually the elements of $\widehat{X_{t}^{\bar{g}}}$ to the closet constellation point may result a catastrophic active index combination that is not included in the reference look-up table, which makes the recovery of index selecting bits impossible [1]. In order to determine the active subcarriers in $\widehat{X_{t}^{\bar{g}}}$ LLR detector is used. The LLR detector of OFDM IM scheme provides the logarithm of ratio of posterior probabilities of the frequency domain symbols by considering the fact that their values can be either non zero or zero. This ratio provides information on active status of corresponding subcarrier index $n$ of transmit antenna t:

$$
\gamma_{t}^{g}(n)=\ln \frac{\sum_{m=1}^{M} p\left(x_{t}^{g}(n)=s_{m} \mid \widehat{X_{t}^{g}}(n)\right)}{P\left(x_{t}^{g}(n)=0 \mid \widehat{X_{t}^{g}}(n)\right)}
$$

\section{Mimo Ofdm Im Using Frequency Offset}

The sensitivity of OFDM systems to frequency offset compared with single carrier System is a major disadvantage. In general, Frequency offset is defined as the difference between the nominal frequency and actual output frequency. In OFDM, the uncertainty in carrier frequency, which is due to a difference in Frequencies of the local oscillators in transmitter \& receiver gives rise to a shift in frequency domain this shift is also referred to as frequency offset.

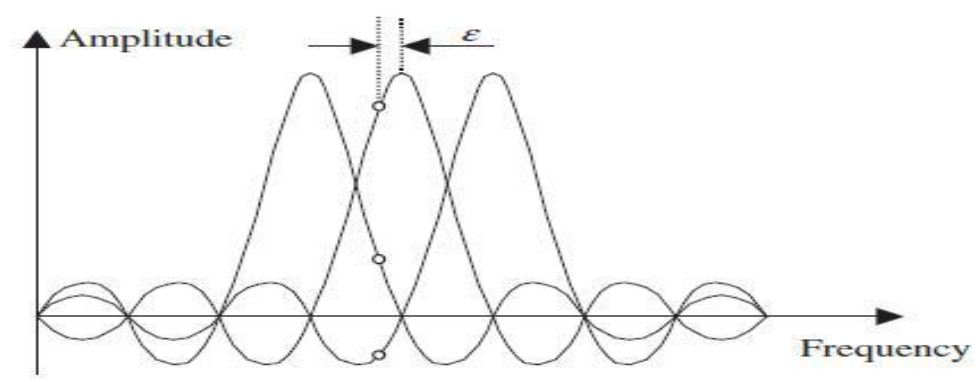

Fig 2: Inter-carrier interference (ICI) subject to CFO.

As shown in Fig2, when the centers of adjacent subcarriers are shifted because of the frequency offset, the adjacent subcarriers nulls are also shifted from the center of the other subcarrier. The received signal contains samples from this shifted subcarrier, leading to ICI. It can also be caused due to the Doppler shift in the channel. The main factor that affects the rate of fading is the mobility of the receiver relative to transmitter. As the receiver moves with some velocity relative to the transmitter, the phase shifts of the received signal changes. This phenomenon is known as the Doppler shift. The Doppler rate gives information about how fast the channel is varying compared to the data rate. In general, Doppler frequency $f_{d}$ is given by: $f_{d}=f_{d \max } \cos \theta$. Where 
$\theta$ is the angle of the signal direction $\& f_{d \max }=\frac{v * f_{c}}{v}, f_{d \max }$ is the maximum Doppler frequency, $\mathrm{v}$ is the velocity of the receiver, $f_{c}$ is the carrier frequency and $\mathrm{c}$ is the propagation velocity of the transmitted signal (speed of light). In this work we propose to use frequency offset with MIMO OFDM IM to overcome this high mobility effect.
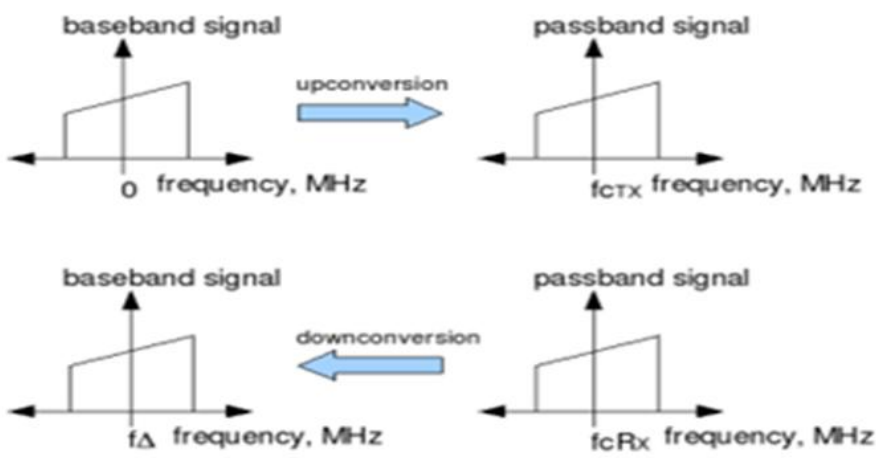

Fig3: concept of frequency offset.

As shown in Fig3, in a typical wireless communication system, the signal to be transmitted is up converted to a carrier frequency prior to transmission. The receiver is expected to tune to the same carrier frequency for down converting the signal to baseband, prior to demodulation. However, due to device impairments the carrier frequency of the receiver need not be same as the carrier frequency of the transmitter. When this happens, the received baseband signal, instead of being centered at DC $(0 \mathrm{MHz})$, will be centered at a frequency $F_{\Delta}$, where $F_{\Delta}=F_{T x}-F_{R x}$. Thus the demodulation of a signal with an offset in the carrier frequency can cause large BER \& may degrade the performance of symbol synchronizer. Therefore important to estimate the frequency offset \& minimizes / eliminates its impact. If frequency offset is denoted as $\Delta \mathrm{fc}$, the OFDM signal generated by the transmitter denoted as $s(t)-(6)$ and $y(t)-(7)$ is the signal received by the receiver, then frequency offset is (8) given by

$$
\begin{gathered}
\mathrm{S}(\mathrm{t})=e^{j w t} \mathrm{X}(\mathrm{t}) \\
\mathrm{Y}(\mathrm{t})=e^{j(w-\hat{w}) t} \mathrm{x}(\mathrm{t}) \\
\Delta_{w}=w-\widehat{w}=2 \pi \Delta \mathrm{Fc}
\end{gathered}
$$

Then received signal has phase offset equal to (9)

$$
\mathrm{Y}(\mathrm{nT})=e^{j \Delta w n T} \mathrm{x}(\mathrm{nT}) \& \emptyset(\mathrm{n})=\Delta w n \mathrm{~T} .
$$

The frequency response of each sub-channel should be zero at all other sub-carrier frequencies, i.e., the sub-channels shouldn't interfere with each other. The effect of frequency offset is a translation of these frequency responses resulting in loss of Orthogonality between the sub-carriers and leading to ICI. These destructive effects of the frequency offset can be corrected by estimating the frequency offset itself \& applying proper correction. In ideal case of no frequency offset, the demodulated value should be zero for whole time. When frequency offset is present, the effect is like random noise which increases with frequency offset. As frequency offset value is increasing, received signal is more distorted and the frequency offset values bigger than $0.6 \%$ the received data are unreadable. Thus in this work we propose that by operating this frequency offset we can reduce the BER (Bit Error Rate) than previous work [1] MIMO OFDM IM.

\section{Matlab Reasults}

In this work, we propose results via numerical calculations on MATLAB platform. We used BPSK (Binary Phase Shift),QPSK (Quadrature Phase Shift Key), QAM (Quadrature Amplitude modulation) modulations \& ML/MMSE detections for three different T*R MIMO configurations: $2 * 2,4 * 4,8 * 8$. The following parameters are assumed in MATLAB i.e. $\mathrm{NFFT}=64, \mathrm{NDSC}=52, \mathrm{nsym}=10^{\wedge} 4 \&$ frequency offset range $= \pm 200 \mathrm{KHz}$. In this work we compare the BER performance of MIMO OFDM IM \& classical OFDM using frequency offset. As seen from figures $(4,5$, and 6$)$ the proposed scheme provides significant BER performance improvement compared to classical OFDM \& still maintains its advantage over classical MIMO OFDM. 


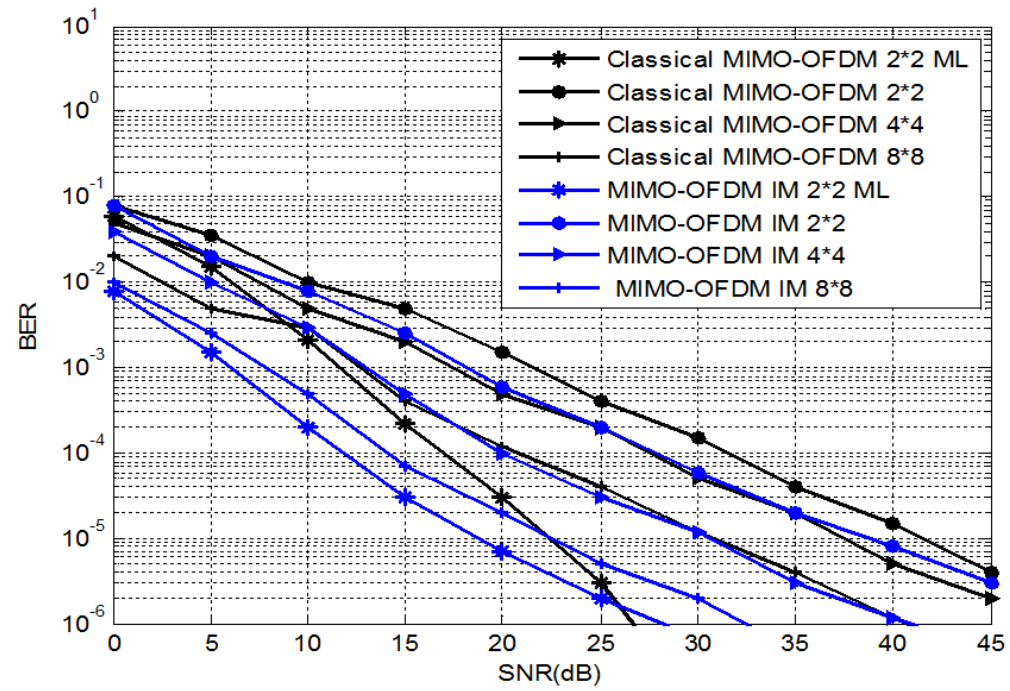

Fig4: Performance comparison of MIMO OFDM \& MIMO OFDM IM using frequency offset for BPSK Modulation, ML/MMSE detection.

Graph plots SNR on $\mathrm{x}$ axis and BER on $\mathrm{y}$ axis. The bit error rate or bit error ratio (BER) is the number of bit errors divided by the total number of transferred bits during a studied time interval. BER is a unit less performance measure, often expressed as a percentage. It achieves significantly better BER (almost nearer to zero) performance than classical MIMO OFDM. This scheme is very useful in wireless communication in order to compensate the effect of mobility. This graph is obtained using BPSK where one binary data is sent at a time. This is the simplest form of modulation which is widely used in communication.

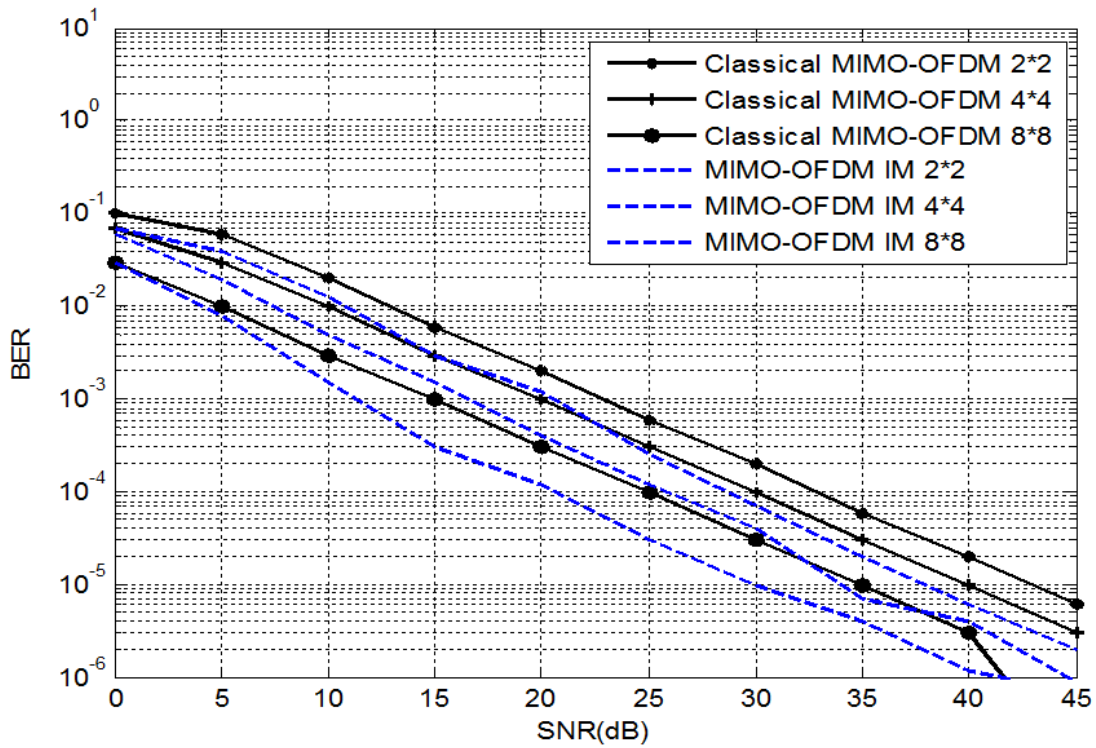

Fig5: Performance comparison of MIMO OFDM \& MIMO OFDM IM using frequency offset for QPSK Modulation, MMSE detection.

As seen from Fig5 it achieves significantly better BER (almost nearer to zero) performance than classical MIMO OFDM. This graph is obtained using QPSK \& it is the digital modulation technique. Quadrature Phase Shift Keying (QPSK) is a form of Phase Shift Keying in which two bits are modulated at once, selecting one of four possible carrier phase shifts $(0, \Pi / 2, \Pi$, and $3 \Pi / 2)$. QPSK perform by changing the phase of the In-phase (I) carrier from $0^{\circ}$ to $180^{\circ}$ and the Quadrature-phase (Q) carrier between $90^{\circ}$ and $270^{\circ}$. This is used to indicate the four states of a 2-bit binary code. Each state of these carriers is referred to as a Symbol. In Fig6 this graph is obtained using QAM, in that same combination of amplitude and phase can carry the concept of transmitting more bits per symbol. 


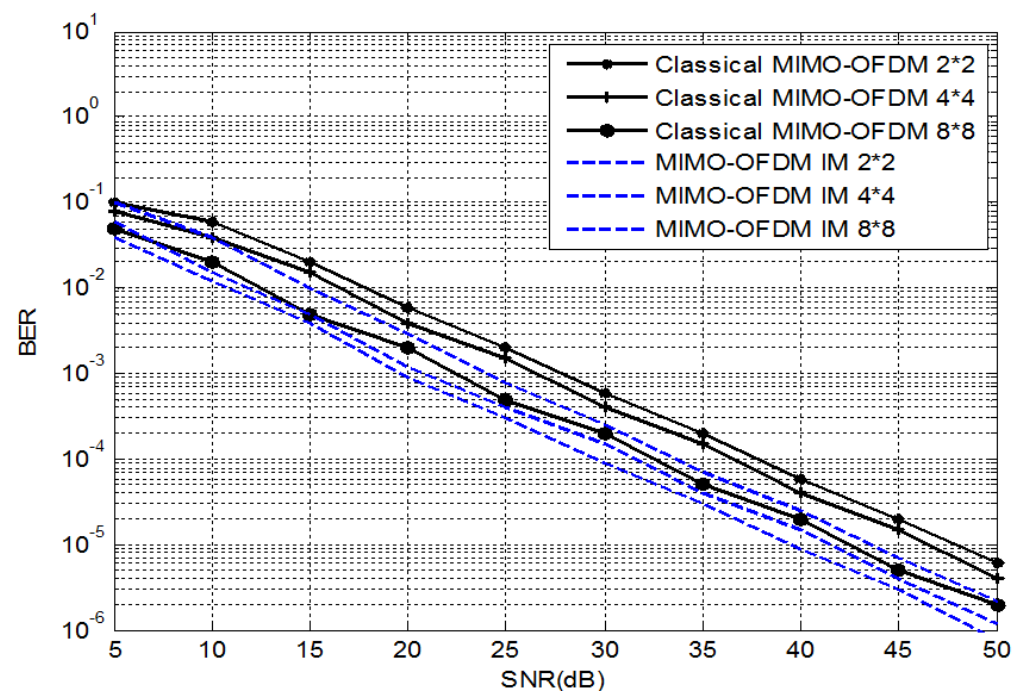

Fig 6: Performance comparison of MIMO OFDM \& MIMO OFDM IM using frequency offset for QAM Modulation, MMSE detection.

\section{Conclusions And Future Work}

The proposed work called MIMO OFDM with index modulation using frequency offset as an attractive alternative multicarrier transmission technique for $5 \mathrm{G}$ networks. It has been shown via numerical calculations on MATLAB platform that proposed work provides significant BER performance improvement almost nearer to zero over classical MIMO OFDM for several different configurations. As research on this concept has been going on in future, we can improve its performance further especially for more complex modulation like QAM by using more advanced detection techniques.

\section{Acknowledgement}

We would like to thank anonymous referees for their valuable comments and suggestions to improve the quality of paper.

\section{References}

[1]. Ertuğrul Başar, Member, IEEE “Multiple-Input Multiple-Output OFDM with Index Modulation”, IEEE Signal Processing Letters, VOL. 22, No. 12, December 2015

[2]. J. Andrews, S. Buzzi, W. Choi, S. Hanly, A. Lozano, A. Soong, and J. Zhang, "What will 5G be?,” IEEE J. Sel. Areas Commun., vol. 32, no. 6, pp. 1065-1082, Jun. 2014.

[3]. E. Başar, Ü. Aygölü, E. Panayırcı, and H. V. Poor, "Orthogonal frequency division multiplexes with index modulation," IEEE Trans. Signal Process. vol. 61, no. 22, pp. 5536-5549, Nov. 2013.

[4]. R. Abu-alhiga and H. Haas, "Subcarrier-index modulation OFDM," in Proc. IEEE Int. Sym. Personal, Indoor and Mobile Radio Commun., Tokyo, Japan, Sep. 2009, pp. 177-181.

[5]. D. Tsonev, S. Sinanovic, and H. Haas, "Enhanced subcarrier index modulation (SIM) OFDM," in Proc. IEEE GLOBECOM Workshops, Dec. 2011, pp. 728-732.

[6]. Y. Ko, "A tight upper bound on bit error rate of joint OFDM and multi-carrier index keying," IEEE Commun. Lett. vol. 18, no. 10, pp. 1763-1766, Oct. 2014

[7]. R. Fan, Y. Yu, and Y. Guan, "Generalization of orthogonal frequency division multiplexing with index modulation,” IEEE Trans. Wireless Commun., no. 99, pp. 1-10, May 2015.

[8]. M. Wen, X. Cheng, and L. Yang, "Optimizing the energy efficiency of OFDM with index modulation," in IEEE Int. Conf. Commun. Systems, Nov. 2014, pp. 31-35.

[9]. W. Li, H. Zhao, C. Zhang, L. Zhao, and R. Wang, "Generalized selecting sub-carrier modulation scheme in OFDM system," in IEEE Int. Conf. Commun. Workshops, Jun. 2014, pp. 907-911.

[10]. E. Başar, "OFDM with index modulation using coordinate interleaving," IEEE Wireless Commun. Lett, no. 99, pp. 1-4, Apr. 2015.

[11]. Y. Xiao, S. Wang, L. Dan, X. Lei, P. Yang, and W. Xiang, “OFDM with interleaved subcarrier-index modulation," IEEE Commun. Lett, vol. 18, no. 8, pp. 1447-1450, Aug. 2014.

[12]. X. Cheng, M. Wen, L. Yang, and Y. Li, "Index modulated OFDM with interleaved grouping for V2X communications," in IEEE Int. Conf. Intelligent Transportation Systems, Oct. 2014, pp. 1097-1104.

[13]. W.Aziz, 1E.Ahmed, G.Abbas," Performance Analysis of Carrier Frequency Offset (CFO) in OFDM using MATLAB," Journal of Engineering (JOE) Vol. 1, No. 1, 2012.

[14]. Pradeep Kumar Nayak, "Inter Channel Interference in High Mobility OFDM," International Journal of Electrical, Electronics and Data Communication, ISSN: 2320-2084 Volume-2, Issue-1, and Jan.-2014.

[15]. Ertuğrul Başar, Member, IEEE "On Multiple-Input Multiple-Output OFDM with Index Modulation for Next Generation Wireless Networks", IEEE Signal Processing Letters, "UNPUBLISHED”. 